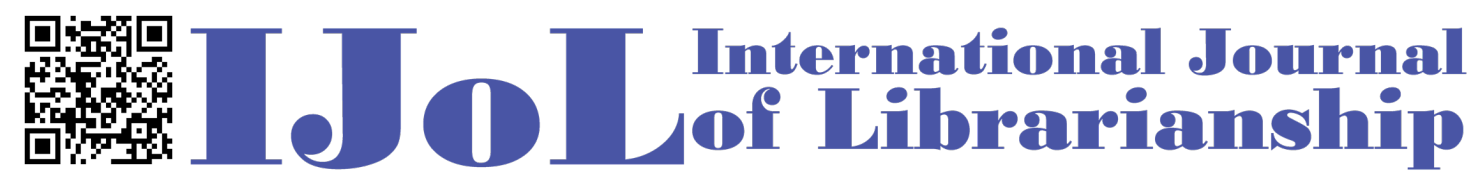

ISSN: 2474-3542 Journal homepage: https://journal.calaijol.org

\title{
How is FOLIO Different from Its Predecessors?
}

Tiewei Liu

\begin{abstract}
:
FOLIO stands for the Future of Libraries is Open and is a future-oriented library service platform. Enlightened by the results of the 2021 International Survey of Library Automation, the author shared thoughts on how FOLIO meets librarians' expectations, why it is time to get involved in the FOLIO project and what challenges FOLIO is facing at the current development stage.
\end{abstract}

To cite this article:

Liu, T. (2021). How is FOLIO different from its predecessors? International Journal of Librarianship, 6(2), 40-48. https://doi.org/10.23974/ijol.2021.vol6.2.207

To submit your article to this journal:

Go to https://ojs.calaijol.org/index.php/ijol/about/submissions 


\title{
How is FOLIO Different from Its Predecessors?
}

\author{
Tiewei Liu, FOLIO Project, Seattle, WA, USA
}

\begin{abstract}
FOLIO stands for the Future of Libraries is Open and is a future-oriented library service platform. Enlightened by the results of the 2021 International Survey of Library Automation, the author shared thoughts on how FOLIO meets librarians' expectations, why it is time to get involved in the FOLIO project and what challenges FOLIO is facing at the current development stage.
\end{abstract}

Keywords: FOLIO, Library Service Platform (LSP), Microservices, Opportunities, Challenges

Libraries are transitioning from their traditional integrated systems to the next generation of library service platforms. The comments collected from the 2021 Survey of Library Automation that was conducted by Marshall Breeding, the well-known library technologist and analyst, show that librarians and system administrators have the following concerns/complaints about their legacy systems and/or the new systems they are moving or have moved to (Breeding, 2021):

- The system or platform is over complicated. The workflow is not user-friendly. There are steep and long learning curves before library staff can use it.

- Problems with upgrades. Some systems require frequent and mandatory updates.

- Frequently introduce features that are half-baked and have errors. Library staff have to spend much time reporting issues.

- Problems with results relevance and accuracy.

- Lack of features, for example, no integration with interlibrary loan, no advanced system to manage requests of print and e-resources and more.

- Lack of flexibility. Need work in the realm of customization and configuration.

- Support is not consistent. Library staff don't always get timely responses from service providers. Quality of the support is not stable, depending on how experienced the supporting staff is. Conflict information might be received. If a third party is involved in the communications, finger pointing happens sometimes.

- Confusing \& Inconsistent terminology in the documentation and software itself. 
- Pricey. Service providers control prices.

From the comments above, we can get a glimpse of librarians' expectations to the ideal library system or platform:

- Simple and easy to use

- Flexibility in implementation, upgrade, customization and configuration

- Full, stable and reliable functionality

- Consistent, responsive and accountable support

- Low cost

Enlightened by the survey results, the author will discuss how FOLIO is fulfilling librarians' needs, why it is a good time to get involved in the FOLIO project and what challenges FOLIO is facing at its current development stage.

\section{WHAT IS FOLIO?}

FOLIO stands for "the future of libraries is open." It is a library services platform, a software development project and a community:

- As an open source library service platform (LSP), FOLIO is built on the microservices architecture and features multi-tenant support, cloud storage, web-based UI, loosely coupled Apps, and one platform for print, electronic and digital resources. The FOLIO LSP is open-ended and can be used on PCs, tablets, iPads, and mobile devices. So far over 40 libraries and library consortia are live with FOLIO LSP and more plan to follow. In fact, the FOLIO LSP has become one of the most competitive library service systems/platforms. The 2021 International Survey of Library Automation shows that "Awareness of the FOLIO library services platform continues to increase with 88 libraries mentioning it among their migration candidates."(Breeding, 2021) FOLIO has become the most competitive product to Alma, which was on the candidates list of 104 libraries.

- As a software development project, FOLIO started in 2016 by the initial partners - EBSCO Information Services, Open Library Environment (OLE) and Index Data. Now it is a single-member limited liability company (SMLLC), a subsidiary of the non-profit organization - Open Library Foundation (OLF). FOLIO is a creative and collaborative effort to deliver a new library services platform to transform library technology. The development is agile, with scrum teams consisting of scrum masters, POs and developers. The project moves forward along the roadmap and under careful capacity planning, which is based on multiple factors, such as feature rankings, feature/bug/tech debt balances, etc. It uses some common practices and utilities of software lifecycle, such as $\mathrm{CI} / \mathrm{CD}$ pipeline, automated test suites, standards around tooling, code coverage expectations, deployment patterns, and established environments. 
Liu / International Journal of Librarianship 6(2)

- As a community, FOLIO boasted its ability to build friendly relationships among all participating institutions and individuals. Now there are over 30 academic libraries (Stanford University Libraries, Cornell University Libraries, etc.) and public libraries (Shanghai Library, etc.) as well as some well-known companies in the FOLIO community. The launch of membership mechanism and the change of its governance model in 2021 substantially turned FOLIO to an open and inclusive community that is managed and built by all the member institutions, whether libraries or companies, and regardless of their sizes, locations and budgets.

\section{HOW DOES FOLIO MEET LIBRARIES NEEDS?}

As a future-oriented product, FOLIO is supposed to meet librarians' expectations for the next generation of smart library service platforms. "FOLIO's platform is built on the idea that library management software should be flexible, modular, extensible, modern, and affordable, and it's gathered a number of partners and contributors to help make that vision a reality." (Watkins, 2018)

Librarians are deeply involved in the entire development lifecycle of FOLIO, which guarantees their needs are fully addressed and the workflows are simple and friendly to users. The FOLIO project brings together professionals from various libraries and service providers so that professionals work together to find the best solution to meet libraries' needs. Product development is based on the actual needs of library staff and their patrons. Users' requirements are well expressed, considered and incorporated in the design, development and testing processes through close communications and interactions with library staff. Therefore, libraries have better understanding of the functionalities and workflows of the platform, ensuring that the product can not only fulfill their functional and non-functional needs, but also is designed to be easy and friendly to use, greatly lowering the learning curves brought by the system conversion and saving the training cost.

FOLIO provides great flexibility in the platform implementation, upgrade, customization and configuration. The FOLIO LSP is built on the microservices architecture. "Microservices are small, autonomous services that work together." (Newman, 2015) Unlike monolithic systems where codebases are usually large and cohesive, yet difficult to develop, deploy and maintain, microservices-based platforms are loosely coupled, with each service focusing on doing one thing well; and that one thing is usually small enough to keep one service independent from another. Services communicate with each other through remote API calls. The isolation of services allows use of different technologies inside each service to achieve better performance. It also allows scaling a particular service and changing a service without affecting other services. With microservices, continuous development, integration and deployment become much easier. Scrum teams to do agile development are also easy to form and can have more deliverables. FOLIO products are released regularly, currently three named releases a year. Information about all the released modules and apps is fully disclosed. Libraries can evaluate whether a certain module/application is ready to use and decide whether to upgrade and upgrade to which version.

FOLIO implementers can still rely on service providers to provide support, just as they do if they use other ILSs and LSPs. Besides, they can actively look for solutions with the assistance from the FOLIO community. As an open-source platform, the FOLIO LSP is transparent, which means its technology is no secret to anyone. The open and collaborative FOLIO community has a 
Liu / International Journal of Librarianship 6(2)

variety of instant communication channels. Therefore, FOLIO implementers no longer just passively wait for their designated service providers to provide support when encountering problems, but have more reliable and timely ways to seek help.

In the procurement of commercial ILSs and LSPs, libraries often cannot do much but passively accept package solutions and high quotations proposed by system or platform providers. Sometimes libraries have to accept unilateral price increases that the providers asked for after they use the product for a certain period. This problem has become even worse with the increasing merger of system providers in the library industry and the reduction of product choices. As an open source service platform, FOLIO provides affordable solutions for libraries. The platform itself can be used for free, but multiple commercial arrangements are available surrounding it. Libraries can deploy and host by themselves, or pay service providers to deploy, support or host for them. In the latter case, service providers can provide one or more services on their own or collaboratively. As multiple companies will provide the same kind of services, libraries can compare different options and have more say in price negotiations.

FOLIO is in the process of fast development. Because of the above reasons, we can reasonably anticipate that the FOLIO LSP can avoid some inevitable problems traditional ILSs and LSPs have and the business models surrounding them. However, it might be too early to say that the FOLIO LSP is mature enough to include all the functions libraries need and all its features work perfectly. In order to build it into an automated service platform with full functionality and high performance that can meet all librarians' expectations, more libraries and companies need to more widely and deeply get involved in the project and collaborate with each other.

\section{WHY SHOULD WE GET INVOLVED?}

It is time to get involved because the FOLIO Project is just beginning its expansion to the next level of LSP services and functionality. Many changes are happening in the FOLIO project. Institutions and individuals all have chances to contribute and shape the future FOLIO.

\section{A. FOLIO HAS BEEN EXPLORING BETTER WAYS TO BUILD AN OPEN COMMUNITY.}

2021 is of great significance to the development of the FOLIO project. Membership mechanism was officially launched. Individual institutions must sign a memorandum of understanding (MOU) with the Open Library Foundation (OLF), the non-profit organization managing the FOLIO project, and commit contributions (monetary or human resources) in order to become a member of the FOLIO community. Though the community meetings and communication channels remain open to all institutions and individuals, only member institutions will have votes in the decision-making bodies of the FOLIO community.

Here is the balance of long-term goals and short-term benefits. FOLIO is undoubtedly at the stage of fast expanding. Capital, manpower and market are all indispensable to its success. The implementation of membership actually created an opportunity for more institutions to make substantial contributions to the project and escalate their involvement to a new level because FOLIO will no longer be sponsored by the few founding partners, rather move toward a real open 
Liu / International Journal of Librarianship 6(2)

community that anyone can participate in and a real open source project built by all. Solid statistics are not available yet to state how the membership will impact the market and if it will reduce participants' desire to join as the new mechanism just started a couple of months ago. However, we can reasonably expect that stable funding and manpower will bring confidence as well as productivity. This optimistic expectation is well supported by at least three facts that happened since the membership model started: 1) the number of libraries adopting FOLIO and announcing plans to adopt FOLIO, and companies signing the MOUs has increased; 2) The August 2021 release is more encouraging compared with the May 2021 release. Statistics showed significant increases in test cases and passing rate as well as decrease in defects identified in the integration environment used for final acceptance and by the Community volunteers; and 3) FOLIO is getting more attention from the world as several new regional interest groups were formed on the wiki run by the FOLIO community.

\section{A. SUBSTANTIAL CHANGES HAPPENED IN THE GOVERNANCE MODEL OF THE FOLIO COMMUNITY.}

The governance model of the FOLIO community was changed and the decision-making bodies were reorganized to be more representative and democratic in 2021.

Before the restructuring, the Stakeholders meeting, consisting of representatives from the original partners of the FOLIO project, was deemed to be the highest decision-making body of the FOLIO community. The Product Council (PC) and the Technical Council (TC) both reported to it, although respectively they routinely made decisions for product- and technology-related issues on their own authority. Members of Stakeholders Meeting, PC and TC were all designated from the original partners of the FOLIO project, that is, EBSCO, Index Data and the Open Library Environment (OLE) libraries.

In the new community structure, however, the Stakeholders Meeting has been replaced by a new body called the Community Council (CC). The CC (9-15 seats), PC (maximum 11 seats) and TC (maximum 11 seats) members are now elected by all the individuals who registered on the formal communication tools Slack, Jira and wiki. Only member institutions can nominate candidates. And each member institution can only have one representative in each council. This new model is obviously more open and inclusive to libraries and companies that didn't have a chance to join in the FOLIO project when it started in 2016. And this model proved to be welcoming in the community judging from the active participation of member institutions in the nominations of candidates and the fact that all the seats of the three councils were filled to their caps. Each council works in the scope of its responsibilities and makes ultimate decisions within that scope. The Community Council fosters a healthy and productive community ecosystem of sustainable collaboration for the FOLIO project. The Product Council maintains the FOLIO feature roadmap to reflect the best interests of librarians and users and to work with the development teams to deliver the roadmap as efficiently as possible. The Technical Council maintains the community's technical policies, best practices, infrastructure, technology roadmap, and the operational health of the FOLIO project. The TC also tracks and reports Technical Debt and facilitates architectural decisions as needed by the community. Though there are no explicit reporting relationships between the $\mathrm{CC}, \mathrm{PC}$ and $\mathrm{TC}$, smooth communications and close collaborations are well established by sending liaisons from one council to another/the other two. 


\section{B. FOLIO PROMOTES THE IDEA OF SYMBIOSIS AND MUTUAL PROSPERITY TO MAXIMIZE WIN-WIN ACHIEVEMENTS.}

FOLIO brings together professionals from libraries and service providers, creates a driving force for innovation, and creates a friendly environment that considers the interests of all participants. Participants of the FOLIO project not only get what they need, but also contribute to the overall progress of the library and information industry.

Through close cooperation and supporting each other with their own strengths, libraries get products that meet their needs, and service providers turn the golden rule of "for the market" into reality. The pluggable mode of microservices brings great flexibility to users. The overall needs of users are fragmented into multiple specific needs. Instead of accepting the entire solution from a single system/service provider as they did before, libraries can now choose one provider for one particular service and another provider for another need. Library service providers can also formulate different product development strategies and form their own unique services for different user groups and their needs.

In addition, FOLIO has greatly improved the relationship between service providers, turning it from competitive to collaborative and complementary. Scrum teams are much easier to form, allowing companies to collaborate on the development of common components for the platform, which elevates the overall quality of FOLIO LSP. This undoubtedly will benefit everyone working on the FOLIO project and using the product. The app store model, which is emerging in community discussions, provides an opportunity and an incentive to innovate through the development of extended apps.

FOLIO not only promotes the collaboration between its participating institutions, but the platform itself also forms a good symbiotic relationship with other library products. The integration of the FOLIO platform with discovery tools, data platforms and ILL systems well demonstrates its ability to develop side-by-side with other products. The Technical Council's efforts to formulate procedures and criteria for accepting external applications and to define the minimal platform well reflect FOLIO's adherence to the idea of openness and inclusiveness.

\section{PARTICIPANTS OF THE FOLIO PROJECT WILL CONTRIBUTE TO THE PROMOTION AND APPLICATION OF NEW TECHNOLOGIES.}

Participants of the FOLIO project will have the opportunity to be involved in the software development lifecycle and apply the new technologies in their own services. The FOLIO Platform is built around a microservices architecture. Key parts of the platform are: Okapi, a proxying API gateway on the server-side; the Stripes framework used to provide a single page UI application in the browser. Behind Okapi, on the server, are the individual microservices in the form of modules. These server-side modules are paired with browser UI modules, which together form specialized applications (or "apps"). Apps may also include Edge modules which allow external systems to integrate into FOLIO (e.g. RTAC, OAI-PMH). (see Figure 1) Unlike traditional integrated library systems which are based-on n-tiered architectures (UI layer, Service Layer, Data Layer), FOLIO's microservices design allows for vertical slices of functionality through Apps which can be added, replaced with alternatives, or removed altogether. 
Liu / International Journal of Librarianship 6(2)

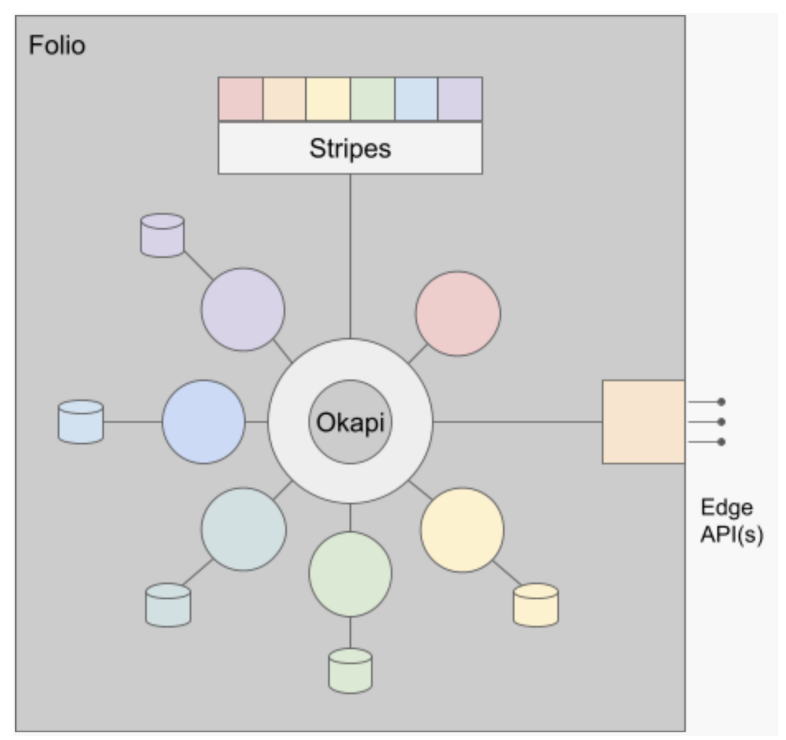

Figure 1. Edge API (s) (provided by Vince Bareau, FOLIO Architect)

The FOLIO LSP includes the FOLIO Platform as well as a suite of apps that are identified to form a consistent solution to everyday tasks of running a library and are developed by the FOLIO core teams and external teams. The Technical Council is working on the value statement and the list of criteria that will allow external code contributions to the FOLIO LSP. The extended apps are provided by FOLIO partners. It's generally encouraged that all FOLIO apps follow the common set of usability and look/feel guidelines. (see Figure 2)

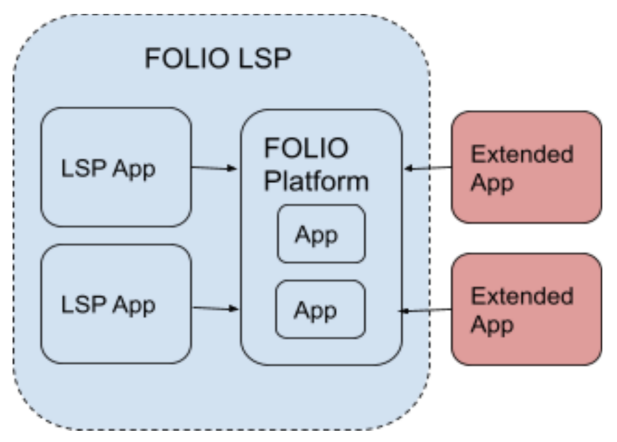

Figure 2. FOLIO Apps (provided by Vince Bareau, FOLIO Architect)

FOLIO embraces an Agile development process, specifically Scrum. FOLIO's scrum teams include scrum master, PO and developers. Currently there are about twenty teams in the FOLIO project. Each team is responsible for a couple of functional areas in library services. Platform wide issues and core functions of the FOLIO LSP are handled by specific teams. Tasks are prioritized for development based on a ranking system focusing on features. Teams use thin thread and try to do one thing well with the development of each feature. A best practice adopted in the project is to align the boundary of each service with the business boundary so that it's obvious to both developers and users what each service does.

The microservices model is very welcoming in the IT industry. In 2021, the IBM Market Development \& Insights conducted a survey with more than 1,200 developers, developer 
Liu / International Journal of Librarianship 6(2)

executives and IT executives currently using a microservices approach, as well as potential users who are exploring or planning to adopt microservices. The results of the survey show that the majority of the survey takers "are excited about the potential of increasing the use of microservices for their organizations" and "believe that the trend will continue".(IBM Corporation, 2021) The IT industry's strong confidence and positive attitude towards microservices will undoubtedly lead to technological innovation and related arrangements promoting this technology, which will also increase people's awareness and strengthen their confidence in microservice-based products. It will be exciting for institutions and individuals to contribute to this historical process by joining the effort of developing and implementing the FOLIO platform.

\section{WHAT ARE THE CHALLENGES?}

FOLIO faces challenges. The biggest concern of libraries is the lack of SysOps professionals who understand microservices-related technologies and can deploy and operate FOLIO. However, due to its flexibility and openness to technologies, microservices are supposed to be easily mastered by developers. The aforementioned IBM survey shows that the talent gap also exists in IT companies. But the survey takers don't think it an insurmountable obstacle. On the contrary, they believe that this gap provides an opportunity to attract talents. We can reasonably expect that, driven by employment opportunities, microservices talents will keep emerging (IBM Corporation, 2021). Even though the majority of libraries don't have microservices expertise internally, they have multiple choices to adopt a microservices based library service platform like FOLIO. Depending on the funding situations, libraries may choose to recruit full-time or part-time system administrators with microservice expertise or train current staff to grow into this role. Small libraries with limited budgets might choose to share technical resources through the consortia they participate in. Alternatively, libraries may use commercial services. In this case, multiple service models can be arranged and multiple service providers are available to choose from.

The FOLIO is not perfect and is still developing. More work is needed to develop missing functions and to improve existing functions to achieve higher performance and stability. Some other topics in hot discussion in the FOLIO community include better defining module boundaries and reducing dependencies; work on documentation to provide consistent and complete documents; automation of testing processes and more. Contribution of ideas and resources and confidence in FOLIO's ability to deliver value are both essential in the process of solving these problems. Involvement also breeds confidence as the FOLIO LSP is becoming more functional and more efficient with each release. Choosing FOLIO is based on rational analysis and judgment. It is a long process to invest in technology and human resources with an eye to the future.

FOLIO uses the Apache License, Version 2.0 in its collaborative and open-source software development. According to the terms of the license, anyone can use, modify and distribute (even for commercial use) the code from the FOLIO project without concern for royalties provided that proper notices are given as required by the license. Such a tolerant rule makes the promotion of FOLIO easier, but also brings convenience to fork code. How to support implementations with a lot of local development and make locally developed features usable to other FOLIO implementers? This is a big challenge to both the FOLIO community and the implementers.

The participants in the FOLIO community vary in many aspects such as locations, cultures, and needs. It usually takes a relatively long time to make decisions because all the opinions need to be fully expressed. At the same time, the Community requires more development capabilities 
Liu / International Journal of Librarianship 6(2)

(coders, POs, sysops, devops, PMs, etc.) as the scope of the FOLIO project becomes bigger and bigger. The huge variety in demands and the limited development capacity make the prioritized development tasks unable to meet the urgent needs of each member library. A good example is that libraries in China prefer artificial intelligence, while their peers in Europe and America have privacy and/or other concerns about the use of AI applications.

In summary, FOLIO has the potential to become one of the dominant library systems and platforms in the near future. Though the FOLIO LSP is not a perfect product yet, the benefits of adopting it are worth the time, effort and investment. It's the best time to join the FOLIO community and get involved in the project because we will all play a role in shaping the next generation of the leading library service platform.

\section{References}

Breeding, M. (2021, March 31). Library Perceptions 2021: Results of the 14th International Survey of Library Automation. Library Technology Guides. https://librarytechnology.org/perceptions/2020/

IBM Corporation. (2021). Microservices in the enterprise, 2021 - Real benefits, worth the challenges: How organizations are finding speed, agility and resiliency through microservices, Results from a survey conducted by IBM Market Development \& Insights. https://www.ibm.com/downloads/cas/OQG4AJAM

Newman, S. (2015). Building microservices. O'Reilly.

Watkins, D. (2018, June 27). Why libraries need to get with apps and APIs. Opensource.com. https://opensource.com/article/18/6/folio

\section{About the author}

Tiewei Liu is the Product Owner of FOLIO China. She had been a digital repository, cataloging and metadata, acquisition and public services librarian at several academic libraries in the U.S. and in China before she joined the FOLIO project. 